\title{
A Study on Epidural Tramadol Compared with Epidural Fentanyl Combined with Low Dose Bupivacaine for the Control of Metastatic Cancer Pain
}

\author{
Resham Bahadur Rana ${ }^{1}$, Zerzina Rahman², Lutful Aziz ${ }^{4}$, AKM Akhtaruzzaman ${ }^{3}, K^{2}$ Kzi Mesbahuddin Iqbal $^{4}$ \\ ${ }^{1}$ Associate Professor, Anesthesiology, Bir Hospital, Kathmandu, Nepal, ${ }^{2}$ Associate Professor, ${ }^{3}$ Professor, Department of Anesthesiology, \\ BSMMU, Dhaka, ${ }^{4}$ Consultant, Anesthesia, Apollo Hospital , Dhaka, Bangladesh.
}

\begin{abstract}
:
Background: Despite advances in the knowledge of pathophysiology of pain and its management, patients continue to suffer from pain in many terminal stage cancer. Tramadol hydrochloride is a weak opioid with analgesic properties, and can be tried for cancer pain management. Objectives: This study was performed to find out the efficacy of the analgesic property of Tramadol through epidural route in cancer patients as an combination with low dose(.125\%) Bupivacaine and to compare with Fentanyl, a $\mu$ opioid agonist. Methods: 50 Cancer patients with or without previous pain management were randomly allocated to one of the two study regime- Group-A (tramadol $50 \mathrm{mg}$ ) and Group-B (Fentanyl $50 \mathrm{mgm}$ ) in combination with .125\% Bupivacaine. Drugs were administered epidurally 6 hourly, 8 hourly and 12 hourly respectively for the $1^{\text {st }}, 2^{\text {nd }}$ and $3 r d$ day. Low dose bupivacaine was added to both groups to enhance quality. Pain scores, blood pressure, respiratory rate, heart rate, side effects and patients' satisfaction score was recorded 6 hourly for 72 hrs. The data yielded from this study were compiled and analyzed by unpaired and paired ' $t$ ' test with $95 \%$ confidence limit. A value of $\mathrm{P}<0.05$ was considered to be significant. $\div$ square test was done for some of the data. Results: Pain scores were significantly decreased in both the groups but were not significantly different. The incidence of side effects including nausea and vomiting was found in both the groups and was not significantly different between the two groups. Conclusion: The use of epidural Tramadol in selected cancer pain patients (especially pain in lower abdomen and lower back) may be very useful and is comparable to opioid in certain situations.
\end{abstract}

Key words: Metastatic cancer pain; Epidural tramadol.

[BSMMU J 2009; 2(2): 66-72]

\section{Introduction:}

Of the estimated nine million new cancer cases every year, more than half is in developing countries and the majority of these patients are incurable by the time their disease is diagnosed ${ }^{1}$. One of the aims of the cancer treatment is to relieve the pain to the patient's satisfaction, so that he or she can function effectively and eventually die free of pain. Commonly pain is caused by a neoplasm or as a complication of cancer therapy ${ }^{2}$. Many epidemiological surveys have concluded that approximately $25 \%$ of patients with localized disease report pain and that the prevalence of pain can be as high as $90 \%$ in patients with advanced cancer $^{3}$. Studies have reported that adequate pain control can be achieved in as many as $88 \%$ of patients with cancer related pain ${ }^{4}$. A recent study used the brief inventory to assess 1308 ambulatory cancer patients and compared their reported pain intensity to the potency of analgesics prescribed to calculate a pain management index. Results showed that $46 \%$ were under managed by

Address for Correspondence: Dr. Zerzina Rahman, Associate Professor (Cardiac Anesthesia), Dept. of Anesthesia, Analgesia and Intensive Care Medicine, BSMMU, Shahbag, Dhaka, Bangladesh. Email: bannya84@gmail.com this standard ${ }^{5}$. It is evident from this study that many patients have inadequate pain management ${ }^{5}$.

Tramadol,(+)cis-2-[(dimethylamino)methyl)]-1-(3methoxyphenyl)-cyclohexanol hydrochloride, is a weak opioid. The analgesic mode of action is not fully understood. It has demonstrable analgesic properties, but its effects are distinct from those of the pure $\mu$ opioid agonist available in clinical practice.

In this study, analgesic efficacy of 'Tramadol' is compared with 'Fentanyl' in the epidural route. Bupivacaine $0.125 \%$ was added to both the drugs. This regime is selected for this study because the delivery of low opioid doses near the sites of action in the spinal cord may decrease supraspinallymediated adverse effects ${ }^{6}$ and in comparison to the neuroablative therapies, epidural opioids have the advantage of preserving sensation, strength and sympathetic function. Furthermore, the addition of a low concentration of a local anesthetic, such as $0.125-0.25 \%$ bupivacaine, to an epidural opioid has been demonstrated to increase analgesic effect without increasing toxicity 7,8,9 .

Epidural fentanyl for analgesia is an established opioid with a short onset of action about 5 minutes and lasting 
for about 2-4 hours with bolus of 25- $100 \mathrm{mg}$ when used alone. When added to $0.125 \%$ bupivacaine, smaller dose of fentanyl will have better pain relief for longer period of time. Furthermore $2-4 \mathrm{mg} / \mathrm{ml}$ of fentenyl in $0.125 \%$ bupivacaine infused epidurally at a rate of 5-20 ml/hr gives good effect in patient with known tolerance to opioids and in patients whose pain was not adequately controlled with epidural opioid alone ${ }^{10}$. Fentanyl, being lipophillic opioid, has less rostral distribution but it is not free from other minor opiate complications.

Furthermore, lack of availability (Controlled), lack of well trained nursing staff and intolerable side effects of fentanyl like respiratory depression ${ }^{11}$, an attempt was made in this study to find out a relatively safer alternative using tramadol. As already mentioned, Tramadol is a weak m receptor agonist and also inhibits reuptake of norepinephrine and promote release of serotonin. Study suggests that they may also have effect through indirect activation of post-synaptic a2 adrenoceptors, blocking impulses reaching the brain ${ }^{12}$. So in this present study a trial was given to find out the effectiveness of epidural tramadol in metastatic cancer pain management. Moreover the study aimed specifically to compare the analgesic effects and the side effects of epidural tramadol with epidural fentanyl as an adjuvant to .125\% bupivacaine and also to find out the cost benefit ratio.

\section{Methods:}

This prospective study was carried out in the department of Anesthesia, Analgesia and Intensive Care Medicine of Bangabandhu Sheikh Mujib Medical University, Shahbag Dhaka. Cancer patients of various organs having pain with or without pain management coming to the 'Kosaka Pain Clinic' of the department had undergone this study. The patients were referred from Onco-therapy department of BSMMU; Cancer Institute, Mohakhali; Dhaka Medical College \& Hospital and from private practitioners. Total 55 patients from both sex with age ranging from 18 years and above were included in the study. They were selected randomly by lottery method and divided in to the following two groups. Group A : consisted of 25 Patients receiving tramadol and Group B consisted of 25 Patients receiving fentanyl. Patients who had pain with metastasis to thorax, lower abdomen, lumbo-sacral vertebrae, pelvis and hip joints were specifically included in the study. Patient not willing to accept the protocol or denied giving written consent and cases with presence of any infection at the spinal site, bleeding diathesis, hypotension, age less than 18 years, patients taking MAO inhibitors and $\mathrm{H} / \mathrm{O}$ epilepsy, bed ridden patients and terminal stage patients( as study was done for 72 hours only) were excluded from the study.
Prior to the study, permission from the ethical committee of BSMMU was obtained. Clinical assessment was done giving emphasis on pain condition and recording the following points- pain at rest, pain on movement, pain during ambulation, and adequate sleep. All patients underwent a full physical examination and were introduced to the Visual Analogue Scale (VAS) that would be used for the appropriate assessment of pain intensity later during the study period. Evaluation of pain was done for 72 hours starting from the time admitted to the either of the study group. Pain situation was evaluated during clinical assessment by Visual analogue scoring system and Verbal Rating Scale scoring system and data were recorded accordingly. Epidural catheters were placed in patients of both groups in the space applicable for the site of lesion. Group A received Tramadol $50 \mathrm{mg}$ (one ampoule=100 mg/ $2 \mathrm{ml}$ ) in $0.125 \%$ bupivacaine ( about $1 \mathrm{ml}$ / spinal segment) and Group B patients received Fentanyl 50 mgm (one ampoule $=100 \mathrm{mg} / 2 \mathrm{ml}$ ) in $0.125 \%$ bupivacaine (about $1 \mathrm{ml} /$ spinal segment) keeping the volume constant in both the groups.

In addition to above study drugs, 50 mg tramadol 6 hourly per oral to all the patients of both groups was given.

The analgesic used by the patient, if any, were stopped and the study regime were started when patient developed pain. The frequency of the drugs was instituted on 6 hourly basis for the first day, 8 hourly in the second day and 12 hourly on third day. The VAS and VRS were recorded at 0 , 05, 15, 30 minutes , hourly and lastly 6 hourly following beginning of the study regime in both the groups. ' 0 ' hour means just before administration of drugs. After study period of 72 hours some patients were continued with epidural catheter and drug regime. Others were followed with standard WHO 3-step analgesic ladder after removal of epidural catheter for further pain management.

After giving the study drugs the haemodynamic and pain parameters were recorded for routine checkup and for evaluation of analgesia. Side effects of the study drugs were also noted down. The systolic and diastolic blood pressures were measured by noninvasive method using an aneroid sphygmomanometer and a stethoscope. The heart rate was measured by palpatory method on radial pulse. Monitor was also used when available in high dependency unit.

Respiratory rate was counted by inspection of chest movement. To measure pain intensity a standard VAS scale measuring 0-10 cm was used. '0' meaning 'no pain' and ' 10 ' being the maximum imaginable pain. For measuring the VRS following numerical were used: $0,1,2,3$ grades for 
'no pain', ‘mild pain', ‘moderate pain' and ‘severe pain' respectively. Needle pin was used to assess the level of sensory effect when affected.

Side effect was recorded primarily by the patient's complaint and also by observation and interrogation to the patient. Use of rescue drug (Diclofenac sodium suppository 50 mg) was recorded when used.The data yielded from this study were compiled and analyzed by unpaired and paired ' $t$ ' test with $95 \%$ confidence limit. A value of $\mathrm{P}<0.05$ was considered to be significant. Non-parametric Chi-Square test was also done in some of the data.

\section{Results:}

Demographic Characteristics of patients are shown in Table I.
A total number of 55 patients were taken in this study. But data was obtained from only 50 patients as two cases had difficulty in epidural catheter insertion and pain measurement was not possible in three patients.

The mean age, weight, height and initial pain scores (VAS and VRS) were comparable in both the groups.

In group-A, organs involved were of uterine cervix 32\%, rectum $24 \%$, Urinary bladder $12 \%$, lung $8 \%$, ovary $8 \%$, gall bladder $4 \%$, and squamous cell carcinoma of skin $4 \%$. In group- $B$, the organs involved were uterine cervix $44 \%$, rectum $24 \%$, Urinary bladder $16 \%$, pancreas $8 \%$ and ovary 8\%. (Data are not presented).

Visual analogue scale and Verbal Rating scales used for pain measurement are shown in figure 1 and 2.

Table I

Patient demography: $(N=25)$

\begin{tabular}{llccc}
\hline Parameter & \multicolumn{2}{c}{ Mean SEM } & PValue \\
\cline { 3 - 4 } & & Group A & Group B & \\
\hline 1 & Age (year) & $45.78 \pm 1.78$ & $44.48 \pm 2.18$ & 0.72 \\
2 & Weight (Kg) & $48.42 \pm 1.17$ & $48.92 \pm 1.45$ & 0.79 \\
3 & Height(Cm) & $161.6 \pm 2.47$ & $160.5 \pm 1.82$ & 0.37 \\
4 & Sex (M:F) & $1.08: 1$ & $0.86: 1$ & \\
\hline
\end{tabular}

$P$ value $<0.05$ is considered significant

Table II

6 hourly Visual Analogue Scores (VAS) in group A and group $B$ with $t$ - test results $\quad(n=25$ each $)$.

\begin{tabular}{lccc}
\hline Time hours & \multicolumn{2}{c}{ Mean \pm SEM } & P value \\
\cline { 2 - 3 } & Group A & Group B & \\
\hline 0 & $7.12 \pm 0.21$ & $6.62 \pm 0.23$ & 0.11 \\
6 & $5.48 * \pm 0.25$ & $5.28 * \pm 0.27$ & 0.59 \\
12 & $4.76 * \pm 0.22$ & $4.84 * \pm 0.26$ & 0.81 \\
18 & $4.12 * \pm 0.27$ & $4.12 * \pm 0.27$ & 1.00 \\
24 & $3.68 * \pm 0.22$ & $4.12 * \pm 0.28$ & 0.22 \\
30 & $3.04 * \pm 0.27$ & $3.44 * \pm 0.27$ & 0.29 \\
36 & $2.92 * \pm 0.26$ & $2.80 * \pm 0.26$ & 0.75 \\
42 & $2.92 * \pm 0.26$ & $2.60 * \pm 0.24$ & 0.37 \\
48 & $2.76 * \pm 0.25$ & $2.44 * \pm 0.20$ & 0.33 \\
54 & $2.56 * \pm 0.20$ & $2.08 * \pm 0.20$ & 0.10 \\
60 & $2.40 * \pm 0.17$ & $1.92 * \pm 0.19$ & 0.07 \\
66 & $2.24 * \pm 0.17$ & $1.80 * \pm 0.17$ & 0.07 \\
72 & $2.04 * \pm 0.17$ & $1.72 * \pm 0.17$ & 0.18 \\
\hline
\end{tabular}

p value $<0.05$ is considered significant $(*=$ Significantly different from ' 0 ' hr. of the same group.

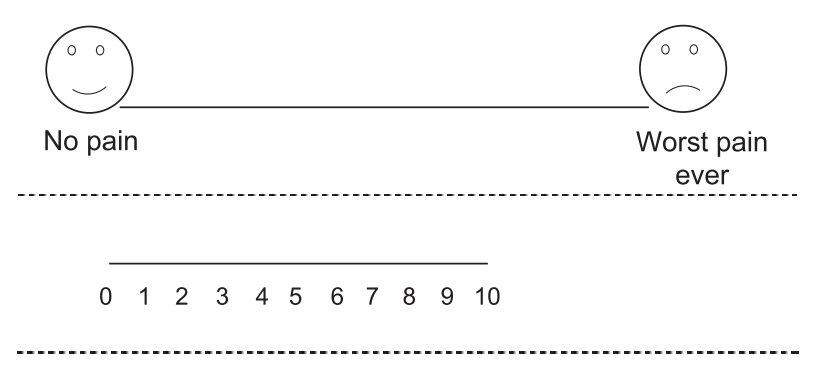

Fig.-1: Visual Analog scale 20

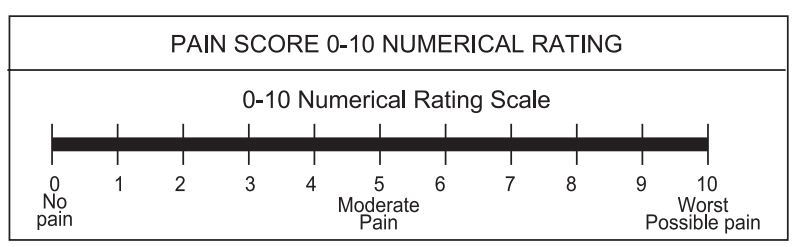

Fig.-2: Numerical rating scale (NRS)/Verbal rating scale (VRS) ${ }^{21}$ 
Visual analogue scores (VAS): The mean \pm SEM values of initial VAS in group A was $7.12 \pm 0.21$ and group B was $6.62 \pm 0.23$. They showed no significant difference between the two groups (Table-III). In both the groups VAS scores decreased following the administration of study drugs. The mean \pm SEM values of VAS in the first $6^{\text {th }}$ hour in group A and B were 5.48 \pm 0.2458 and $5.28 \pm 0.274$ respectively. The paired ' $\mathrm{t}$ ' test within the groups showed significant differences. Following study drugs intervention the mean values of VAS obtained 6 hourly in group A and group B were also described in Table II. The results of ' $\mathrm{t}$ ' test between the groups were not significant in any interval.

\section{Table III}

Number of patients with specific side effects in each group $(n=25)$

\begin{tabular}{lccc}
\hline Side effects & Group A & Group B & P value \\
\hline Nausea & 3 & 2 & 1.00 \\
Vomiting & 1 & 0 & 1.00 \\
Pruritus & 1 & 2 & 1.00 \\
Constipation & 2 & 2 & 1.00 \\
Motor block & 0 & 2 & 0.49 \\
Urinary retention & 0 & 0 & \\
\hline
\end{tabular}

$P$ value $<0.05$ is considered significant

Verbal Rating Scores (VRS): The mean \pm SEM values of VRS in group A and B before intervention of study drugs were $2.88 \pm 0.00$ and $2.92 \pm 0.00$. There was no significant difference between the two groups. The 6 hourly mean values of VRS after drug intervention in group $A$ and group $B$ is given below in Figure 3 . The 6 hourly VRS values at $42^{\text {nd }}$ hour onwards in both the groups were $0.96 \pm 0.00$. The value obtained at $36^{\text {th }}$ hour and $42^{\text {nd }}$ hour were significantly different between the two groups. Rest of the values didn't show any statistical significance between the two groups. However statistical significance was obvious within the groups right after the use of first dose in both the groups. The linear comparison of the 6 hourly comparative statistical results between the two groups is shown in Figure 3.

Frequency of side effects is shown in table III. During the study period, side effects were also recorded whenever complained by patient or observed during the study period. Nausea being one of the common side effects of both the drugs was found in both groups. 3 patients in group A and 2 patients in group $B$ were found to have it. Vomiting was noted in 1 (one) patient in group A but none in group 6hrly Verbal Rating Scores in group A and Group B with $P$ value

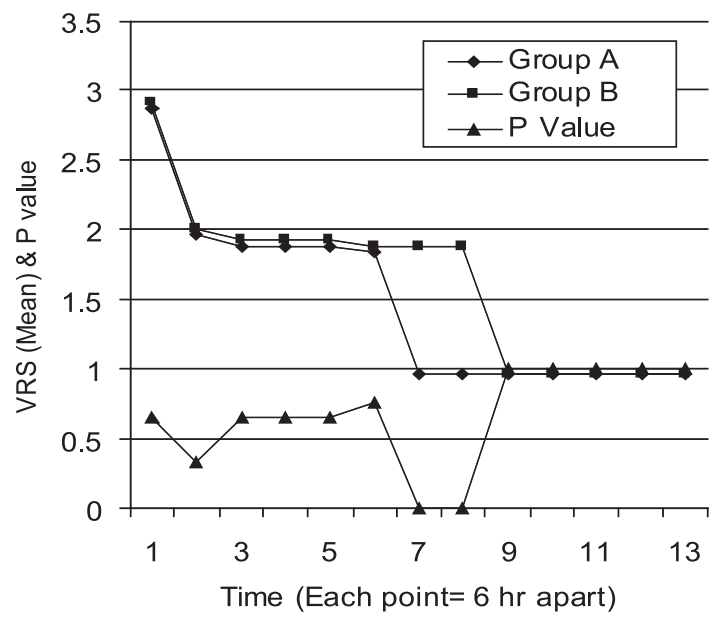

$\mathrm{p}$ value $<0.05$ is considered significant * = Significantly different from ' 0 ' hr. of the same group.(Value at ' 0 ' hour means is before the study drug ).

Fig.-3: 6hrly Verbal rating Scores (VRS) in group A and group $B$ with $t$ - test results ( $n=25$ each)

B. Similarly, pruritus was found in one (1) patient in group A and 2 patients in group B. Pruritus in group A was so severe that it required treatment with antihistamine. Constipation was found in 2 patients in each group. Motor block was observed in two patients of group B. Chi square test was done for comparison of side effects between two groups and found to be insignificant statistically.

Patients' satisfaction was evaluated according to the operational definition ( Score 1- Very satisfied; Score 2Quite satisfied; Score 3- Neither satisfied nor unsatisfied; Score 4- Unsatisfied; Score 5- Very Unsatisfied) at the end of study period in both the groups by direct verbal interrogation. In group A, 5 patients were 'very satisfied';

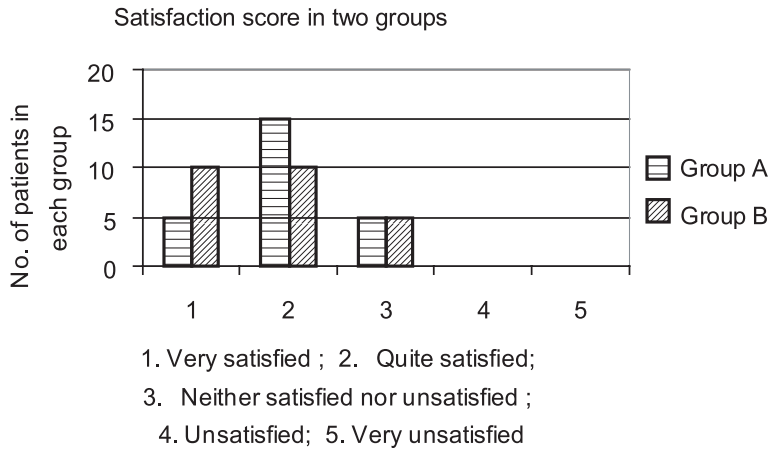

Fig.-4 : Number of patients with type of satisfaction in each group with $\div$ square test values $(n=25)$ 
15 patients were 'quite satisfied'; and 5 patients were 'Neither satisfied nor unsatisfied'. In group B, 10 patients were 'very satisfied'; 10 patients were 'quite satisfied'; and 5 patients were 'Neither satisfied nor unsatisfied. No matter whatever their VAS or VRS score and side effects, none of the patient was in 'unsatisfied' or 'very unsatisfied' category in both the groups. Figure 4 shows the satisfaction comparisons.

\section{Discussion:}

Effective pain control is essential for optimal care in cancer patients. However, despite advances in the knowledge of pathophysiology of pain, and more effective techniques of pain management, patients continue to suffer from considerable pain in many cancer situations especially in terminal stages. A multidisciplinary approach is always necessary and encouraged for optimum pain control and should be appreciated. The treatment of the pain in cancer patients is primarily palliative. The aim of pain management are to relieve/reduce pain, improve mobility and function. Although cancer therapy can contribute towards effective pain relief, an invasive treatment is often required to improve the quality of life in these patients. Thus, the pain management in cancer patients requires a multidisciplinary approach for a successful treatment eg involvement of oncologist, surgeons, psychologists, anesthesiologists etc.

This study was an attempt to find out the efficacy of the analgesic property of Tramadol through epidural route in cancerous patients. Addition of low dose Bupivacaine was used in an attempt to get a synergistic effect with Tramadol. A comparison was made with Fentanyl, which is widely and effectively used in many painful situations.

Strong opioids like morphine and fentanyl are the gold standard drugs for pain management through various routes of administration in the patients with moderate to severe pain. Morphine is cheap and is also available in oral forms in other countries but are not readily available in Bangladesh (Controlled drugs). Same factors are also true for the availability of intravenous fentanyl. Moreover we don't have sufficient skilled manpower and equipment to observe and manage their side effects like respiratory depression, if any.

The contribution of enhanced monoaminergic transmission to the analgesic actions of tramadol is supported by the blocking of antinociceptive effects of intrathecal tramadol by yohimbine and ritanserin. These á2-adrenoceptor antagonists do not block the antinociceptive effects of morphine. These findings supports that tramadol achieves monoaminergic spinal modulation of pain through indirect activation of postsynaptic á2-adrenoceptors, blocking impulses reaching the brain.

This study was aimed to see whether Tramadol could be used in unmanaged metastatic cancer pain. Tramadol, a WHO ladder II drug (weak opioid) is currently available in Bangladesh.

So in this study, the efficacy of pain relief was compared with fentanyl and side effects like respiratory depression and haemodynamic changes were evaluated.

The epidural route was chosen to reduce the dose of the drug thereby reducing the side effects as well as to have more specific spinal segmental effect for pain management. Combination of low dose of bupivacaine is again to reduce the dose of the drug and to get a synergistic effect and to reduce the side effects of the drugs.

Not many studies have come out regarding epidural Tramadol for pain managemen. All of them have encouraging results.

Chrubasik and Magora ${ }^{13}$ in a study compared $100 \mathrm{mg}$ Tramadol with $3 \mathrm{mg}$ of morphine epidurally and found it to be equianalgesic. Another study ${ }^{14}$ compared $100 \mathrm{mg}$ tramadol and lignocaine with $4 \mathrm{mg}$ of morphine and lignocaine for post-operative analgesia. The number of the patients were only 20 (10 in each group). The analgesia was equally effective and long lasting in both groups.

In one study, Delikan et al ${ }^{15}$ compared epidural tramadol $50 \mathrm{mg}$, tramadol $100 \mathrm{mg}$ and bupivacaine $0.25 \%$ for postoperative pain. The entire study regimes were significantly effective to relieve the pain. Tramadol produced more relief of pain than the other two, which was statistically significant $(\mathrm{P}<0.05)$. Their VAS score was 7.4 before starting the drug in group B (tramadol $100 \mathrm{mg}$ ) which came down to 2.3 by 24 hours. The dose was not required before 6 hours in many patients, but some of them required only one or two doses in 24 hours.

Analgesic effect of both drugs was well noted in both the groups. The VAS decrease was statistically significant with the very first doses in both the groups. This study approaches very near to Delikan et $\mathrm{al}^{13}$ study with tramadol $100 \mathrm{mg}$.

In this present study both the groups had similar VAS score at ' 0 ' hour and were not significantly different statistically. There was significant decrease of VAS within the groups $(\mathrm{P}<0.000)$. But there was no significant difference between the groups. The VRS response to the study drugs was similar to the VAS response in both the 
groups. Overall decrease in VAS score was statistically significant in both the groups with ' 0 ' hours of each group. This significant analgesic effect with $50 \mathrm{mg}$ tramadol may be due to synergistic effect achieved by the addition of $0.125 \%$ of bupivacaine. Moreover the 6 hourly dose schedule given for the first day gave significant pain relief.

Baraka et $\mathrm{al}^{14}$ (1993) found decrease in mean $\mathrm{PaO} 2$ in morphine group but none in tramadol group with an increase in PaCO2 which was difficult to interpret .

Delikan et a ${ }^{15}$ did not find any respiratory depression effect with either of the tramadol doses.

Vickers et $\mathrm{al}^{16}$ conducted a study on tramadol giving emphasis on its respiratory depression effect. They compared morphine $(0.143 \mathrm{mg} / \mathrm{kg})$ with three different doses of tramadol $(0.5 \mathrm{mg} / \mathrm{kg}, 1 \mathrm{mg} / \mathrm{kg}$, and $2 \mathrm{mg} / \mathrm{kg})$ in postoperative patients of lower abdominal surgeries. All the drugs were given via intravenous route. The study showed that increasing dose of tramadol was responsible for decrease in respiratory rate. But this decrease by the maximum dose of tramadol was less than that of morphine. It was statistically significant between tramadol $2 \mathrm{mg} / \mathrm{kg}$ and morphine $0.143 \mathrm{mg} / \mathrm{kg}$.

Robert-Jan et al ${ }^{17}$ studied the efficacy and safety of tramadol versus morphine for moderate and severe postoperative pain with special regard to respiratory depression. They found significant decrease in transcutaneous $\mathrm{SaO} 2$ in morphine than in tramadol $(\mathrm{p}=0.0101)$.

In this present study only respiratory rate was recorded during study period. There was no significant difference between the two groups at ' 0 ' hour. Following the intervention of the drugs the respiratory rate (RR) was relatively constant in group A but the RR was decreased in group $\mathrm{B}$. The decrease was significant in the group $\mathrm{A}$ with ' 0 ' hour. Moreover, the decrease in RR obtained after $3^{\text {rd }}$ dose till last doses between the two groups were statistically significant. The preservation of the RR by tramadol may be due to its analgesic effect other than ì agonist activity.

Recorded data shows that there was some fluctuation on heart rate (HR) in both the groups. Although there is no statistically significant difference between the groups throughout the study period, there seems to be more tendencies to decrease HR in group B. The pharmacological property may be responsible for its cause. The data suggests that none of the drug have consistent effect on HR in such painful situations.
Before administration of drugs the blood pressure was comparable and the difference was not significant statistically between group A and group B. However, after intervention of the study drugs, decrease in mean arterial pressure (MAP) was so less as clinically not important and may be due to addition of bupivacaine.

Other side effects like nausea, vomiting, pruritus, constipation were also recorded in few cases in both the groups. Data of side effects found in both groups were not significant statistically. All these side effects are common to both of these drugs.

Sedation produced by the drugs was also recorded. Patients with sedation were graded into five different categories from awake and alert to unresponsive to painful stimulus. Patients were found to have sedation in first three categories in both the groups. But the value did not differ significantly between the two groups.

In a recent study, epidural administration of tramadol through the PCA method following gynecologic cancer surgery was found to be a more effective analgesia in lower doses when compared to the intravenous administration ${ }^{18}$.

In another recent study, on the basis of their experience and review of the literature it was decided that there is definitely a place for weak opioids in the treatment of moderate cancer pain ${ }^{19}$. One of the most interesting and useful weak opioids is tramadol. Its unique mechanism of action, analgesic efficacy and profile of adverse reactions have been the reason of performing many experimental and clinical studies with tramadol in this study ${ }^{19}$.

Finally, the satisfaction was categorized into five different categories. Data was recorded in comparable numbers in both the groups. Numbers of patients in each category were statistically insignificant between the groups. Neither of the group had unsatisfied or very unsatisfied patients. Looking to availability of the study drugs, fentanyl was not always readily available in the market. Again, it is more expensive than the other. Even if available, patient has to pay more because of its higher price and frequency of required dose. Tramadol on the other hand is widely available in the market of Bangladesh and is less expensive. We have a large field left for future research on different approaches of administration of epidural tramadol in cancer pain management, such as continuous low dose infusion of tramadol in 'walking epidural' .

\section{Conclusion:}

From this study it may be concluded that tramadol may be alternatively used epidurally to manage the chronic 
metastatic cancer pain in appropriate patients. Moreover, tramadol is equally effective (in the compared doses) and also safe to use in the cancer pain as an adjuvant to .125\% bupivacaine. Other advantages of tramadol include easy availability, cheaper cost and less respiratory depression.

\section{Acknowledgement:}

This study was based on a original clinical study done for thesis work in the department of Anesthesia, Analgesia \& Intensive Care Medicine of Bangabandhu Sheikh Mujib Medical University during September 2000 to November 2001.It was completed by the author and was approved by a board of examiners in 2002 .

\section{References:}

1. http://whqlibdoc.who.int/publications/9241544821.pdf.

2. Payne R, Weinstein SM, Hill CS. Assessment and management of pain. In:Levin VA (Ed). Cancer in the Nervous System. New York: Churchill Livingstone, 1996, p 448.

3. Jacox A, Carr DB, Payne R. New clinical practice guidelines for the management of cancer pain. N Engl J Med 1994a; 330: p 169-173.

4. Zech DFJ , Grond SUA, Lynch J, Herterl D, Lehmann KA. Validation of World Health Organization Guideline for cancer pain relief: a 10 year prospective study. Pain 1995; 63: p 6576 .

5. Cleeland CS, Gonin R, Hatfield AK, et al. Pain and its treatment in outpatients with metastatic cancer. N Engl J Med 1994: 330:p 592-596.

6. L. Brian Ready, Acute Pain.Anesthesia Vol 2, Fourth Edition 1994 ; p 2327-2344.

7. Du Pen S L, Ramsay H; Compounding local anesthetics and narcotics for epidural analgesia in cancer outpatients. Anesthesiology,1988; 69: p 247-248.

8. Nitescu , Appelgren L, Linder L E et al 1990; Epidural versus intra-thecal morphine-bupivacaine assessment of consecutive treatment in advanced cancer pain. Journal of Pain and Symptom Management.5:p 18-26.
9. Sjoberg M, Appelgren L, Einarsson S et al; Long term intrathecal morphine and bupivacaine in 'refractory' cancer pain.Results from the first series of 52 patients. Acta Anaesthesiologica Scandinavica ;1991.

10. Cullen ML, Staren ED, EL-Ganzour A et al: Continuous epidural infusion for analgesia after major abdominal operation: a randomized, prospective, double blind study, Surgery $98: \mathrm{p}$ 718, 1998 .

11. Adams A P \& Pybus D A 1978. Delayed respiratory depression after use of fentanyl during anaesthesia. British Medical Journal,1, p 278.

12. D J R Duthie. Remifentanil and tramadol: British Journal of Anesthesia 1998; 81: 51-57. 1998

13. Chrubasik J , Magora F. Relative analgesic potencies of epidural opiates in treatment of acute pain. Pain 1990; Suppl 5: S154.

14. Baraka A, Jabbour S, Ghabssh M, Nader A, Khoury G, Sibai A. A comparison of epidural tramadol and epidural morphine for post-operative analgesia. Can J Anesth 1993; 40: p 308-313.

15. .Delikan AE, Vijayan R. Epidural tramadol for post-operative pain relief. Anesthesia 1993; 48:p 328-331.

16. M.D.Vickers , M Morgan, P.S. J. Spencer, M.S. Read. Drugs in anesthetic and Intensive Care Practice, eight edition, 1999.

17. Robert-Jan M. Houmes, Voets MA, Verkaaik A, et al.1992. Efficacy and safety of tramadol versus morphine for moderate and severe post-operative pain with special regard to respiratory depression. Anesthesia and Analgesia 74: p 510514.

18. Yavuz L, Eroglu F, Ozsoy M; The efficacy of intravenous versus epidural tramadol with patient-controlled analgesia (PCA) in gynecologic cancer pain ; Eur J Gynaecol Oncol ; 2004;25(2): p 215-8;

19. Wojciech Leppert, Jacek uczak; The role of tramadol in cancer pain treatment-a review ; Journal of supportive care in cancer; 0941-4355 ; vol 13, no. 1; p 5-17.

20. Visual analogue pain scale. Available from http://www.bjmp.org/ files/wasch2007/bjmp0309daabis.jpg

21. Verbal rating scale. Available from http://www.nature.com/ nrrheum/journal/ $\mathrm{v}_{3} / \mathrm{nII} /$ images/neprehum0646-iz.jpg 